\title{
Hippocampal subfields predict positive symptoms in schizophrenia: First evidence from brain morphometry
}

\author{
S Kühn ${ }^{1,2}$, F Musso $^{3}$, A Mobascher ${ }^{4,5}$, T Warbrick $^{5}$, G Winterer ${ }^{5,6}$ and J Gallinat ${ }^{1}$
}

\begin{abstract}
Alterations of hippocampal anatomy have been reported consistently in schizophrenia. Within the present study, we used FreeSurfer to determine hippocampal subfield volumes in 21 schizophrenic patients. A negative correlation between PANSSpositive symptom score and bilateral hippocampal subfield CA2/3 as well as CA1 volume was found on high-resolution magnetic resonance images. Our observation opens the gate for advanced investigation of the commonly reported hippocampal abnormalities in schizophrenia in terms of specific subfields.
\end{abstract}

Translational Psychiatry (2012) 2, e127; doi:10.1038/tp.2012.51; published online 12 June 2012

\section{Introduction}

Alterations of hippocampal anatomy have consistently been reported in schizophrenia. ${ }^{1}$ It has been hypothesized that resulting memory problems lead to increases of illusory pattern completion and are therewith involved in the generation of psychotic symptoms due to dysfunctional association forming. ${ }^{2}$ In particular, the so-called autoassociative learning mechanism within the subfield cornu ammonis (CA) 3 together with CA1 allows for rapid binding of events that cooccur. Postmortem studies have reported reduced size and altered dendritic arborization of CA3 pyramidal neurons, predominantly in patients with positive symptoms. ${ }^{3}$ However, the role of hippocampal CA subfields volume for positive symptoms has not been investigated in vivo.

\section{Methods}

We studied 21 schizophrenic patients (mean age $=34,2 \pm 8.2$ years; five female; number of episodes $=2.5 \pm 2.0$; age of onset $=26.4 \pm 7.8$ years; 19 medicated with atypicals) giving written informed consent with the Positive and Negative Syndrome Scale (PANSS). ${ }^{4}$ Hippocampal subfields volume was assessed fully automatic with FreeSurfer $r^{5}$ on the average of 2-4 (mean $=3.4 \pm 0.9)$ T1-weighted magnetic resonance images (3T Siemens Trio; MPRAGE, resolution $1 \times 1 \times 1 \mathrm{~mm}^{3}$ ). The computational model by van Leemput ${ }^{5}$ incorporates a prior distribution that makes predictions about where neuroanatomical labels are expected to occur. This prior is based on a generalization of various probabilistic atlases, and is automatically learned from manual segmentations of the hippocampal formation in MRI images. A likelihood distribution then predicts how the labeled image, on which each voxel is assigned a unique neuroanatomical label, translates into an individual's MRI image. We focussed on the volume of the entire hippocampus, CA1, CA2/3, CA4/dentate gyrus, subiculum and presubiculum, disregarding fimbria and hippocampal fissure, because the later two are the smallest subfields that are considerably less reliably segmented ${ }^{5}$ and disregarding the so-called 'hippocampus' subsegment that contains mainly the tail of the hippocampus where subfields were not discernable.

\section{Results}

We observed a negative Pearson's correlation between PANSS-positive symptom score $(M=12.43$, s.d. $=4.27)$ and bilateral hippocampal subfield CA2/3 volume $(r(21)=-0.46$, $P<0.05)$ as well as CA1 volume $(r(21)=-0.44, P<0.05$, Figures 1 and 2, Table 1), indicating that patients with stronger positive symptoms have smaller CA2/3 and CA1 subfields. No other subfield showed significant correlations neither with the Positive Syndrome score $(P>0.26)$ nor with the Negative Syndrome score $(M=16.05$, s.d. $=4.74, P>0.42)$. Although the results do not survive conservative Bonferroni correction for multiple testing, the fact that not only bilateral CA2/3 and CA1 but also right and left structures separately survive statistical thresholding with $P<0.05$ is remarkable. The subfield volumes do not correlate with chlorpromazine equivalents of neuroleptic medication (CA2/3: $r(21)=0.04, P=0.86$; CA1: $r(21)=0.00, P=0.99)$.

\section{Discussion}

The results are compatible with models of hippocampal CA3 processes interacting with CA1 in the generation of positive symptoms. ${ }^{2}$ These hippocampal subfields have been suggested to act as a binding module for cortical circuits containing weakly related sensory representations. CA3 in particular has been proposed to create representations of space and time as a basis of conscious awareness. ${ }^{6}$

\footnotetext{
${ }^{1}$ Charité University Medicine, St Hedwig-Krankenhaus, Clinic for Psychiatry and Psychotherapy, Berlin, Germany; ${ }^{2}$ Department of Experimental Psychology, Ghent Institute for Functional and Metabolic Imaging, Ghent University, Ghent, Belgium; ${ }^{3}$ Department of Psychiatry, University of Düsseldorf, Düsseldorf, Germany; ${ }^{4}$ Department of Psychiatry, University of Mainz, Mainz, Germany; ${ }^{5}$ Institute of Neurosciences and Medicine, Helmholtz Research Centre Jülich, Jülich, Germany and ${ }^{6}$ Cologne Center for Genomics, University of Cologne, Cologne, Germany

Correspondence: Dr S Kühn, Faculty of Psychology and Educational Sciences, Department of Experimental Psychology, Ghent Institute for Functional and Metabolic Imaging, Ghent University, Henri Dunantlaan 2, 9000 Gent, Belgium.

E-mail: simone.kuhn@ ugent.be

Keywords: CA1; CA2/3; FreeSurfer; hippocampus; schizophrenia; volumetry

Received 23 January 2012; revised 18 April 2012; accepted 21 April 2012
} 

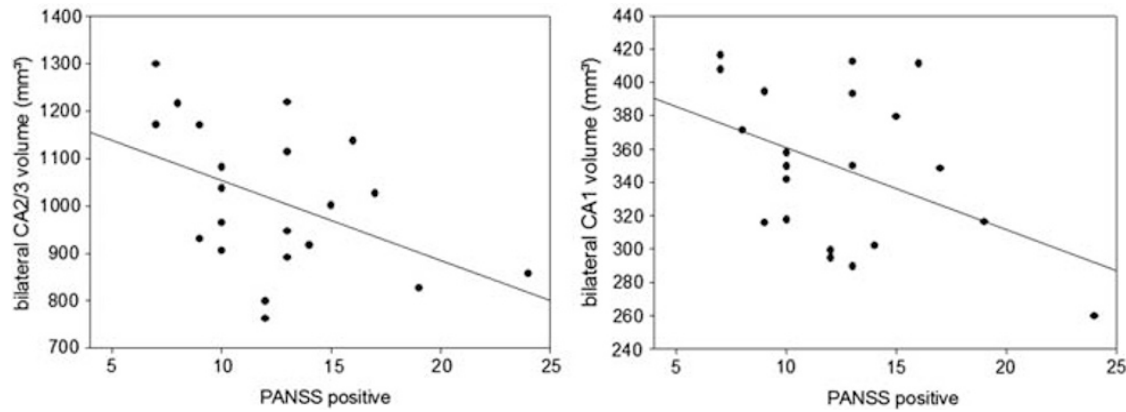

Figure 1 Negative correlation between the positive symptom subscale of the Positive and Negative Syndrome Scale (PANSS) and bilateral hippocampal CA2/3 and CA1 subfield volume.
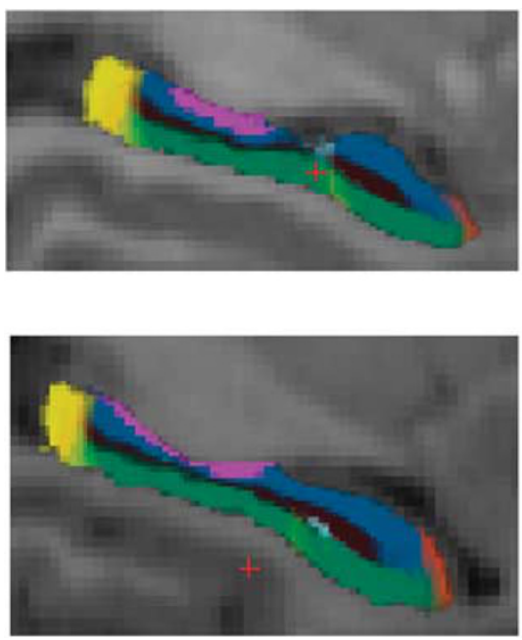

Figure 2 Left hippocampal subfield segmentation of two exemplary subject.

Table 1 Mean \pm s.d. of bilateral larger hippocampal subfield volumes (in $\mathrm{mmt}$ ) and its correlation with Positive and Negative Syndrome Score

\begin{tabular}{|c|c|c|c|}
\hline $\begin{array}{l}\text { Hippocampal } \\
\text { subfields }\end{array}$ & $\begin{array}{c}\text { Mean } \\
(s . d ., \text { in } \\
\left.\text { mm }^{3}\right)\end{array}$ & $\begin{array}{c}\text { Correlation with } \\
\text { positive symptoms } \\
\text { (Pearson's } \\
\text { correlation } \\
\text { coefficient) }\end{array}$ & $\begin{array}{c}\text { Correlation with } \\
\text { negative symptoms } \\
\text { (Pearson's } \\
\text { correlation } \\
\text { coefficient) }\end{array}$ \\
\hline $\begin{array}{l}\text { Whole } \\
\text { hippocampus }\end{array}$ & $7010(817)$ & -0.26 & -0.07 \\
\hline $\mathrm{CA} 1$ & $350(46)$ & $-0.44^{\star}$ & 0.03 \\
\hline $\mathrm{CA} 2 / 3$ & 1013 (149) & $-0.46^{\star}$ & -0.07 \\
\hline $\begin{array}{l}\text { CA4, dentate } \\
\text { gyrus }\end{array}$ & $563(78)$ & -0.20 & -0.08 \\
\hline Subiculum & 669 (73) & -0.13 & -0.19 \\
\hline Presubiculum & $453(40)$ & -0.19 & -0.11 \\
\hline
\end{tabular}

*Indicates correlations with $P<0.05$.

A subfield dysfunction would integrate sensory representations abnormally resulting in positive symptomatology; for instance, hallucinations. ${ }^{2}$ Furthermore, it has been suggested that the disinhibition of hippocampal regions can stimulate hyperdopaminergic states and therewith produce psychosis. ${ }^{7}$

Our observation opens the gate for advanced investigation of the commonly reported hippocampal abnormalities in schizophrenia in terms of specific subfields. However, replication of results is needed in a larger sample of unmedicated patients with further differentiation of $\mathrm{CA} 2 / 3$ volumes. Furthermore, a limitation of the present study is the
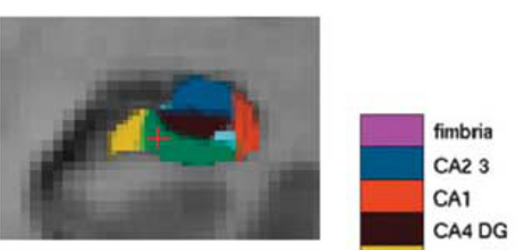

CA4 DG presubiculum subiculum

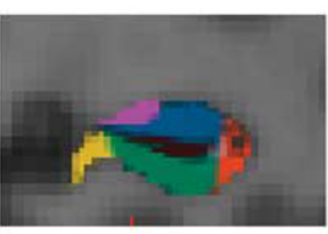
hippocampal fissure hippocampus inf. lateral ventricle choroid plexus

relatively low, albeit common, resolution of $1 \times 1 \times 1 \mathrm{~mm}^{3}$. Future studies should consider using a sequence with a higher spatial resolution.

\section{Conflict of interest}

The authors declare no conflict of interest.

1. Klär AA, Ballmaier M, Leopold K, Häke I, Schaefer M, Brühl R et al. Interaction of hippocampal volume and $\mathrm{N}$-acetylaspartate concentration deficits in schizophrenia: $\mathrm{A}$ combined MRI and 1H-MRS study. Neuroimage 2010; 53: 51-57.

2. Tamminga $C A$, Stan AD, Wagner AD. The hippocampal formation in schizophrenia. Am J Psychiatry 2010; 167: 1178-1193.

3. Kolomeets NS, Orlovskaya DD, Rachmanova VI, Uranova NA. Ultrastructural alterations in hippocampal mossy fiber synapses in schizophrenia: A post-mortem morphometry study. Synapse 2005; 57: 47-55.

4. Kay SR, Fiszbein A, Opler LA. The positive and negative syndrome scale (PANSS) for schizophrenia. Schizophr Bull 1987; 13: 261-276.

5. Van Leemput K, Bakkour A, Benner T, Wiggins G, Wald LL, Augustinack J et al. Automated segmentation of hippocampal subfields from ultra-high resolution in vivo MRI. Hippocampus 2009; 19: 549-557.

6. Behrendt RP. Contribution of hippocampal region $\mathrm{CA} 3$ to consciousness and schizophrenic hallucinations. Neurosci Biobehav Rev 2010; 34: 1121-1136.

7. Lisman JJE, Coyle J, Green RW, Javitt DC, Benes FM, Heckers S et al. Circuit-based framework for understanding neurotransmitter and risk gene interactions in schizophrenia. Trens Neurosci 2008; 31: 234-242.

Translational Psychiatry is an open-access journal published by Nature Publishing Group. This work is licensed under the Creative Commons Attribution-Noncommercial-No Derivative Works 3.0 Unported License. To view a copy of this license, visit http://creativecommons.org/licenses/by-nc-nd/3.0/ 